\title{
Capital social estructural y educación, un estudio desde la perspectiva de Pierre Bourdieu: estudio de caso Universidad $\mathbf{E C C I}^{3}$
}

\author{
Manuel Fernando Cabrera Jiménez4 \& Claudia Liliana Infante Rincón5 \\ Universidad ECCI, Colombia
}

Recibido, agosto 03 de 2015

Concepto evaluación, diciembre 14 de 2015

Aceptado, enero 27 de 2016

\begin{abstract}
Referencia: Cabrera Jiménez, M.; Infante Rincón, C. (2016). "Capital social estructural y educación, un estudio desde la perspectiva de Pierre Bourdieu: estudio de caso Universidad ECCI". Revista Academia y Virtualidad, 9, (1), 105-122
\end{abstract}

\section{Resumen}

Este estudio se desarrolló con el fin de identificar el nivel de capital social estructural en el contexto educativo de la Universidad ECCI. El trabajo se desarrolló con base en el planteamiento de una hipótesis sobre una revisión teórica centrando su atención en los postulados de Pierre Bourdieu y otros referentes teóricos destacados en el tema. Se utilizó una metodología de corte descriptiva con un enfoque cuantitativo con dos variables: estudiantes matriculados a partir de tercer semestre y docentes de planta, vinculados a la institución desde 2013. Se diseñó un instrumento para la recolección de la información, compuesto por tres dimensiones: el capital social cognitivo, el capital social estructural y la inclusión. El objetivo de este artículo es dar cuenta de los resultados obtenidos en la dimensión estructural del capital social caracterizada por el perfeccionamiento de relaciones normalizadas que potencialicen estrategias orientadas a fortalecer la asociatividad. La investigación permitió concluir que los niveles de capital social estructural en la Universidad ECCI presentan una tendencia media baja, lo cual se puede considerar una deficiencia para la construcción de capital social, instancia que demanda un plan estratégico que potencialice y se asuma como objetivo colectivo para el bienestar.

Palabras clave: educación, capital social, asociatividad, estructura social, redes.

\footnotetext{
${ }^{1}$ Artículo de investigación científica y tecnológica como resultado del proyecto de investigación "Medición de capital social Universidad ECCI 2015", aprobado institucionalmente.

${ }^{2}$ Magíster en docencia, docente investigador de la Escuela Colombia de Carreras Industriales - ECCI, e-mail: mcabreraj@ecci.edu.co

${ }^{3}$ Magíster en docencia, docente investigador de la Escuela Colombia de Carreras Industriales - ECCI, e- mail: cinfanter@ecci.edu.co
} 


\title{
Structural social capital and education. An analysis supported by Pierre Bourdieu principles: A case study at Universidad ECCI
}

\begin{abstract}
This study was developed in order to identify the level of the structural social capital in the educational environment of the Universidad ECCI. The work was based on a hypothesis from a theoretical review focusing on principles by Pierre Bourdieu and other prominent theorists concerning the subject. A descriptive methodology was applied using a 2-variable quantitative approach -1) enrolled students from third semester, and 2) Faculty teachers linked to the institution since 2013. We designed a 3-dimension tool for data collection, i.e. cognitive social capital, structural social capital and inclusion. The aim is to report the results of the structural dimension of social capital characterized by improving normalized relations to trigger strategies aimed at strengthening the associativeness concept. The research concluded that levels of structural social capital at ECCI show a low average trend, which can be considered as a failure to build social capital, an event that demands a strategic plan to trigger the welfare but as a mutual goal.
\end{abstract}

Keywords: education, social capital, associativeness, social structure, networks.

\section{Capital social estrutural e educação, um estudo desde a perspectiva de Pierre Bourdieu: Estudo de Caso Universidade ECCI}

\section{Resumo}

Este estudo de caso desenvolveu-se a fim de identificar o nível de capital social estrutural no contesto educativo da Universidade ECCI. O trabalho desenvolveu-se com base na formulação de uma hipótese sobre uma revisão teórica centrando sua atenção nos postulados de Pierre Bourdieu e outros referentes teóricos destacados no tema. Utilizou-se uma metodologia de tipo descritiva com uma abordagem quantitativa com duas variáveis: estudantes inscritos a partir do terceiro semestre e docentes titulares, vinculados à instituição desde 2013. Desenhou-se um instrumento para a coleta da informação, composto de três dimensões: o capital social cognitivo, o capital social estrutural e a inclusão. O objetivo deste artigo é de dar conta dos resultados obtidos na dimensão estrutural do capital social caraterizada pelo aperfeiçoamento de relações normalizadas que potencializem estratégias orientadas a fortalecer a associatividade. A pesquisa permitiu concluir que os níveis de capital social estrutural na universidade ECCI apresentam uma tendência média baixa, o qual pode se considerar uma deficiência para a construção do capital social, instância que demanda um plano estratégico para potencializar e se assumir como objetivo coletivo para o bem-estar.

Palavras chave: educação, capital social, associatividade, estrutura social, redes. 


\section{Introducción}

Desde la década de 1980, varios autores han realizado investigaciones que giran en torno al capital social. Pierre Bourdieu basa su concepto en el estudio de las redes y la interacción social a partir de la acumulación de recursos reales y potenciales, como fuente de relaciones institucionalizadas que permiten reconocimientos mutuos. En este artículo se analizará la postura de Pierre Bourdieu a partir de reflexionar en algunos de sus referentes teóricos destacados, con respecto a las redes, el capital simbólico, tipos de capital, privilegios y educación, elementos que se articulan en la dimensión del capital social estructural.

Asimismo, los investigadores centraron su atención en la revisión de otros referentes teóricos destacados orientados a la construcción del capital social como un constructo del siglo XX, en las ciencias sociales. Entre ellos se destacan, Coleman, (1988), aborda el capital social desde la perspectiva del estructuralismo y el sentido social de la educación. Según Putnam, elementos como la confianza, la consolidación de redes y la administración pública, son fuentes de capital social; Woolcock, de igual forma, tiene en cuenta los organismos supranacionales como el Banco Mundial, los valores y las relaciones horizontales, indica que la normatividad es fuente de capital social, mientras que Francis Fukuyama aborda el capital social desde la cultura y la confianza y la familia principalmente.

La investigación realizada para la generación de este artículo, se sustenta en la fundamentación teórica referida anteriormente, la cual se contempló como principal fuente para la realización de los instrumentos diseñados para el trabajo de campo, el cual tuvo como contexto la comunidad académica de la Universidad ECCI, docentes y estudiantes, vinculados a partir del segundo semestre del 2013. Es pertinente aclarar que el estudio se realizó durante el primer semestre del año 2015 con estudiantes y docentes vinculados con una antigüedad en la institución mínimo de tres semestres académicos.

Este proyecto tiene como objetivo central identificar estrategias desde un diagnóstico endógeno sobre capital social que permita, el fortalecimiento de nuestra colectivo en función de fortalecer la capacidad asociativa de la comunidad académica, e identificar el nivel de reconocimiento que hacen las personas de las normas, los niveles de confianza y reciprocidad propios del capital social en el contexto de la Universidad ECCI.

\section{Marco referencial}

Pierre Bourdieu estudia las tensiones al interior de las clases sociales y las diferencia entre estas, basadas en la disponibilidad y acceso a los diferentes tipos de capital, por lo cual, asume que las relaciones sociales generadas a través de las posiciones son las que importan y trascienden, sobreponiendo una perspectiva objetivista sobre una subjetivista; en este sentido, sus aportes cobran sentido en las diferentes estructuras sociales y en la particularidad de cada una de ellas. Un factor relevante en el estudio de las relaciones del capital cultural basado en la relación de capital social y educación es la desigualdad, la cual está directamente relacionada con la estratificación social; asimismo, con los modelos de jerarquización y el acceso al poder lo cual obedece a la evolución de las elites en el poder siguiendo a Bourdieu (1998), demostrando que aquellos sujetos que no adquieren un nivel de formación impuesto por la cultura dominante serán considerados, excluidos del sistema.

En ese sentido, es un privilegio acceder a la educación, ya que las personas que pueden ingresar y hacer uso de ésta, se les facilita posteriormente ostentar y ejercer poder; de igual forma, puede llegar a disponer de mejores recursos frente a los individuos excluidos del sistema educativo. Este postulado es abordado por Bourdieu, el cual le permite reconocer el capital social como una estructura organizada 
por niveles. Asimismo, hace un análisis que trasciende la vida social fáctica, al considerar que los sujetos aunque son individuos sociales, cuentan con autonomía afectada por determinaciones individuales, es decir el habitus.

Según Bourdieu, el capital social coexiste con el capital económico y el capital cultural, es decir, este escenario se puede interpretar como contexto para que algunos individuos o clases sociales acumulen poder o lo transformen en diferentes tipos de capital. En otras palabras, el capital social para Bourdieu no es ajeno al desarrollo de otros tipos de capital, a su vez, tiene gran relación con el desarrollo económico, ya que sus análisis parten de una concepción sociológica que plantea con base en la identificación de las características sociales de los agentes, en función de las necesidades y las relaciones que de allí surgen con base en su accionar histórico y cultural como sociedad.

(Bourdieu, 1988), reconoce la educación como el medio que transforma las personas, brindándoles oportunidades de interactuar a nivel individual y colectivo. En tal sentido, estas relaciones demandan capital social. Asimismo, las personas que acceden a la educación transforman su capital social y, en esa misma medida, obtienen mayores posibilidades y aspiraciones. En este sentido, el capital social es el resultante del gozo de la utilización y posesión de los recursos existentes y potenciales que se dispone para hacer parte de un grupo reconocido por una red socialmente institucionalizada.

En este sentido, el capital social se legitima a partir de la interacción con otros capitales. De esta forma, las personas buscan hacer parte de redes en lo posible homogéneas, que garanticen la interacción con sujetos similares y generar posibles contactos por ejemplo pertenecer a un club social o acceder a la educación en determinada universidad y permite generar contactos que fortalezcan sus redes de apoyo.
La teoría de los campos sociales, propuesta por Bourdieu a mediados del siglo XX, plantea que todas las sociedades se pueden considerar espacios donde emergen estructuras diferenciadas a partir de la distribución de las formas de poder o de capital que dan sentido a la estructura social.

Para Bourdieu, el campo es el espacio de juego o interacción de los participantes constituidos como organizaciones con reglas propias y agentes, quienes se asumen como actores dinámicos que pueden crear, luchar y participar, por intermedio de la acción, manifestadas en estrategias, alianzas llevadas al campo de lucha para lograr la obtención del capital simbólico que otorga legitimidad, prestigio y autoridad al agente que lo posee.

Según Pierre Bourdieu, existen diferentes campos (Bourdieu, Razones prácticas sobre la teoría de la acción): el económico, el lingüístico, el educativo, donde cada uno de ellos cuenta con sus propias instituciones y reglas específicas de funcionamiento. Es decir, cada campo cuenta con una estructura autónoma que permite la interacción de acuerdo con la posición o rol de dominado o dominante de los agentes que lo conforman, con base en las normas propias de cada campo. Por ejemplo, las personas que estudian adquieren mayor poder en la sociedad y podrían asumir con facilidad el rol de dominante, pues el conocimiento empodera a las personas, les hace cambiar su visión prospectiva, eleva el autoestima, entre otros.

En la obra de Bourdieu, las relaciones de poder son una de las constantes variables de reflexión, por ello aborda el concepto "violencia simbólica", concepto que emerge como resultado del análisis de las sociedades modernas desde la educación. Esta noción de "violencia simbólica" surge con base en los estudios realizados sobre educación en Francia, junto a Jean Claude Passeron, cuyo análisis fue planteado en la obra Reproduction (1970), en la cual se desarrollan los conceptos teóricos de una noción de "violencia simbólica". 
Ahora bien, según el autor (Bourdieu, 2000) el término violencia simbólica es diferente de la violencia física o sicológica; en otras palabras, es la lucha conjunta por lograr los objetivos o metas propuestas. Entendiendo esta lucha como la suma de esfuerzos por lograr los propósitos, metas u objetivos individuales o colectivos. Por ejemplo, en el caso de los investigadores científicos, todo el grupo de investigación trabaja en pro de validar la hipótesis, presentar los resultados propios de la investigación, pero su objetivo colectivo es ser reconocidos y obtener visibilidad.

Por otra parte, es de reconocer que los diferentes tipos de violencia: simbólica, física y sicológica, tienen diferentes representaciones en el contexto social, pero comparten el cuerpo como el escenario, el cual se asume como instrumento del habitus, porque parte de una imposición de un acontecimiento arbitrario sin consentimiento de todas las partes involucradas soportado en un bagaje cultural y consolidado histórico, que permite un reconocimiento y modo de actuar propio de ser y le permite comprender el mundo desde el rol que ejerza como dominado o dominante.

En toda relación entre dominado y dominante, se puede evidenciar la violencia simbólica, la cual obtiene legitimidad y reconocimiento, a partir del mismo desconocimiento de su condición propia de violencia por las partes.

A la hora de dar forma, en La Reproduction (1970), al concepto de "violencia simbólica", Bourdieu recurre a una peculiar articulación de las herramientas proporcionadas por Marx, Durkheim, y Weber, de tal modo que entre los tres se establece una especie de corrección recíproca. La violencia simbólica, que opera en el plano del capital simbólico, es por ello relativamente autónoma respecto a la violencia física ligada a la posesión de otras formas de capital (político, militar, económico, etc...). (Vázquez, 2002, pág. 151).

En síntesis, el capital social para Bourdieu se genera desde las posibilidades que se crean en el interior de las estructuras sociales, constructo que se ve moldeado por el habitus, el campo y la violencia simbólica, la cual se presenta como un elemento generado con el permiso de los actores sociales, dado que se crean relaciones entre agentes a partir de entender el habitus como un sistema de prácticas de producción entre agentes sociales, y el campo como el escenario de interacción social, correlación que permite la consolidación de estructuras que surgen desde un esquema de pensamiento y acción de los agentes actuantes que se han interiorizado a través del tiempo de su existencia.

El capital social para Bourdieu se asume como un conjunto de relaciones que potencializan desde otros capitales el accesoal poder, mediado por la educación como factor transformador de sociedades y paradigmas del contexto local y externo del sujeto, permitiendo a las personas al interior de las estructuras sociales asumir un rol de dominante o dominado -en muchos casos inconscientemente- según el capital social disponible. Bourdieu distinguió tres clases fundamentales de capital: económico, cultural y social. (Fernandez\&Ferrerias, 2009, pág. 35).

En este sentido el capital social estructural fundamentado en el respeto a las normas, la confianza, y la reciprocidad elementos reconocidos por Putnam, Coleman y Fukuyama, se asumen como valores generados desde capital social cognitivo, los cuales posibilitan alto nivel de incremento de beneficio a la sociedad que se organiza en redes, ya que el factor que diferencia cada organización no es un obstáculo para aislar sino que permite integralidad, desde esta perspectiva disminuyendo la visibilidad de las diferencias lo que evidencia el aislacionismo. Igualmente, cada una de las dimensiones sustenta una serie de componentes que se comparten y permiten evidenciar una integralidad que le dan sentido al capital social como constructo. Esta integralidad se fundamenta en compartir principios valores los cuales constituyen cimientos sólidos de los procesos de asociatividad, desarrollo de vida cívica y consolidación de la confianza, 
elementos que se ven reflejados en el nivel y número de redes cívicas formales e informales que consolidan los miembros de una comunidad.

\section{Articulación de la dimensión estructural con el capital social}

La integralidad de las dimensiones le brindan al concepto de capital social, una perspectiva holística que en la práctica le permite a los grupos sociales acceder cómo gestionar recursos con bajos costes de transacción, integrándose en redes en acciones que serían restringidas en otra dinámicas por fuera del capital social, generadas como consecuencia de las tensiones con base en las diferencias de las estructuras sociales y en los procesos de estratificación preestablecidos.

Finalmente, se presenta una concepción de capital social construida desde la articulación de los elementos anteriormente analizados por cada uno de los teóricos que han abordado su estudio, en tal sentido se puede indicar que el capital social es el constructo social que se genera, a partir de la interacción entre organizaciones a nivel horizontal y vertical, conformadas por personas que representan intereses colectivos, con motivaciones individuales, y características culturales y sociales específicas, que se integran por medio de la asociatividad en redes formales e informales que tienen como objetivo potencializar la cooperación y la reciprocidad a partir de identificar las normas establecidas, generando interacción entre sociedad civil y gobierno, que potencialice relaciones basadas en valores, confianza y solidaridad en coherencia con la cultura endógena, favoreciendo el bienestar económico y social.

\section{Metodología}

Para este ejercicio, se definió un tipo de investigación descriptiva con enfoque cuantitativo, el cual permite verificar el logro de los objetivos propuestos que dan origen a la investigación que sustenta este artículo; asimismo, el proceso investigativo está basado en un estudio de caso, el cual se caracteriza por delimitar un contexto o una población específica asumido como una sola unidad de análisis. En este sentido, se aborda el estudio de capital social, en el contexto de la Universidad ECCI, a partir de la identificación de dos variables y el estudio de su correlación definida así: Variable 1, educación superior en el contexto de la Universidad ECCI y una segunda variable denominada, capital social. La variable Educación Superior se comprende para este trabajo como el conjunto de estudiantes y profesores que se hayan vinculado a la institución como mínimo desde 2013, característica que conlleva a poder contar con un conocimiento experiencial de las relaciones en el interior de la universidad en el año 2015, cuando se realizó el estudio.

La variable capital social está comprendida a partir del proceso cognitivo del individuo quien interioriza y apropia como constructo colectivo social los valores, las normas y los principios institucionales, los cuales, les permiten establecer buenas relaciones en su entorno educativo para lograr así el desarrollo desde la asociatividad, como comunidad articulada bajo unos mismos principios.

En términos de Bourdieu, la educación es el medio que transforma a las personas, brindándoles un contexto nutrido de oportunidadesparainteractuaranivelindividual y colectivo. Asimismo, tienen la posibilidad de incrementar relaciones que demandan capital social, logrando así, potencializar el trabajo en equipo, la asociatividad, el respeto por el otro, la libre expresión y el bienestar de la comunidad educativa en general. A partir de este constructo, los investigadores plantean la siguiente hipótesis, la educación superior es el principal ente promotor de transformación en la sociedad, ya que su principal rol es aportar para la transformación positiva de una comunidad, ya que una sociedad con un buen nivel de educación, reconoce las normas y los valores comúnmente aceptados, así como identifica su capacidad de aporte y responsabilidad a la 
sociedad que pertenece. Reconociendo de esta forma el capital cultural que se asume como el componente que da forma y sentido a un grupo social. En este escenario, el capital social cumple una función de potencializar -desde la asociatividad- la construcción de relaciones perdurables, a partir del reconocimiento de los componentes que dan sentido al capital social. En este sentido, este artículo, con base en la investigación realizada, pretende validar la importancia de las relaciones generadas en el capital social en el contexto de la Universidad ECCI, en la ciudad de Bogotá.

Esta hipótesis cobrará sentido en el contexto de la Universidad ECCI, si al concluir el estudio ésta arroja un valor $\mathrm{p}$ o valor de probabilidad $<=0.05$ de reconocimiento y construcción de capital social en la comunidad ECCI, resultado que se presentará en las conclusiones del estudio.

\section{Diseño muestral}

Para este ejercicio, se determinó como población objeto del estudio la comunidad académica total de la Universidad ECCI en modalidad presencial pregrado (estudiantado y planta docente); restringida a los estudiantes matriculadosque esténcursando tercersemestre en adelante, y docentes que hayan trabajado con la institución por al menos un año. Como se sabe, cada una de las subpoblaciones está en un constante crecimiento, y la población de estudio también crece proporcionalmente a éste; para ello, haremos uso de la teoría de muestreo, con el fin de poder representar correctamente la población.

Teniendo en cuenta estas características de la población ECCI, así como la intencionalidad de los objetivos del estudio planteados en la investigación y los referentes teóricos sobre capital social principalmente desde Pierre Bourdieu, en primer lugar se diseñó el instrumento, para la recolección de la información. Éste se estructuró a partir de definir tres grandes dimensiones, por una parte el capital social cognitivo, con 11 ítemes en segundo lugar el capital social estructural con 12 ítemes y en tercer lugar la inclusión con 10 ítemes.

Para efectos de este artículo, se socializarán los resultados de la dimensión estructural, sustentada desde la importancia que Pierre Bourdieu le brinda a las redes, consideradas éstas por el autor como base para la consecución de privilegios, como bien lo cita en sus primeros trabajos sobre el tema en la obra La reproducción (1970). Posteriormente, estos conceptos se van refinando y se van constituyendo como capital simbólico concebido como la justificación por la cual, las personas luchan al interior de una sociedad y disfrutan de los beneficios que éste genera. En este sentido, el capital social estructural se asume como la capacidad de los individuos para movilizar sus recursos y relaciones de capital en función de las características propias de un entorno específico institucionalizado fundado en la pertenencia.

En segundo lugar, se realizó la validación y evaluación del instrumento lo que llevó a realizar algunos ajustes en términos de tipo de pregunta la determinación de algunos parámetros de interés que inciden directamente sobre la fórmula del tamaño de muestra, todo con la ayuda de una muestra piloto.

Para este proceso, la muestra piloto fue aplicada a veinte estudiantes de tres programas académicos, y a cinco docentes del área de investigación. Ambos procedimientos no evidenciaron problemas en el instrumento (entendible y medible), y nos permitieron identificar el tamaño de muestra necesario para efectuar la recolección de datos (Monroy, 2012).

En tercer lugar, con los datos obtenidos de las estadísticas oficiales de la universidad se logró deducir que el tamaño del estudiantado que entraría como población objetivo eran 11000 estudiantes. La definición del tamaño de muestra necesario se encuentra en las siguientes tablas, se encuentra en color 
amarillo; la primera tabla indica el número 20\%, pues corremos el riesgo de que no todos mínimo de estudiantes a encuestar; la segunda los estudiantes encuestados respondan. tabla incluye el factor de "no respuesta" de

\begin{tabular}{|l|l|l|l|l|}
\hline $\mathrm{N}$ & \multicolumn{1}{l}{11000} \\
\hline $\mathbf{0 , 2 2 7 5}$ & Em & $\mathbf{0 , 0 2 5}$ & $\mathbf{0 , 0 5}$ & $\mathbf{0 , 1}$ \\
\hline 2,24 & $97,50 \%$ & 1566 & 438 & 113 \\
\hline 1,96 & $95 \%$ & 1241 & 339 & 87 \\
\hline 1,645 & $90 \%$ & 904 & 241 & 61 \\
\hline 1,44 & $85 \%$ & 706 & 186 & 47 \\
\hline 1,28 & $\mathbf{8 0} \%$ & 566 & 147 & 37 \\
\hline
\end{tabular}

\begin{tabular}{|l|l|l|l|}
\hline Em & $\mathbf{0 , 0 2 5}$ & $\mathbf{0 , 0 5}$ & $\mathbf{0 , 1}$ \\
\hline $\mathbf{9 7 , 5 0 \%}$ & $\mathbf{1 8 8 0}$ & $\mathbf{5 2 6}$ & $\mathbf{1 3 6}$ \\
\hline $\mathbf{9 5 \%}$ & $\mathbf{1 4 8 9}$ & 407 & 104 \\
\hline $\mathbf{9 0 \%}$ & $\mathbf{1 0 8 5}$ & $\mathbf{2 8 9}$ & $\mathbf{7 3}$ \\
\hline $\mathbf{8 5 \%}$ & $\mathbf{8 4 8}$ & $\mathbf{2 2 3}$ & $\mathbf{5 6}$ \\
\hline $\mathbf{8 0 \%}$ & 679 & 177 & 45 \\
\hline
\end{tabular}

En el caso de los docentes, la situación fue la siguiente:

\begin{tabular}{|l|l|l|l|l|}
\hline $\mathbf{N}$ & \multicolumn{2}{l|}{744} \\
\hline $\mathbf{0 , 1 8}$ & Em & $\mathbf{0 , 0 2 5}$ & $\mathbf{0 , 0 5}$ & $\mathbf{0 , 1}$ \\
\hline 2,24 & $97,50 \%$ & 491 & 243 & 81 \\
\hline 1,96 & $95 \%$ & 445 & 202 & 63 \\
\hline 1,645 & $90 \%$ & 381 & 154 & 46 \\
\hline 1,44 & $85 \%$ & 331 & 124 & 36 \\
\hline 1,28 & $80 \%$ & 289 & 102 & 28 \\
\hline
\end{tabular}

\begin{tabular}{|l|l|l|l|}
\hline Em & $\mathbf{0 , 0 2 5}$ & $\mathbf{0 , 0 5}$ & $\mathbf{0 , 1}$ \\
\hline $\mathbf{9 7 , 5 0 \%}$ & $\mathbf{5 8 9}$ & 292 & 97 \\
\hline $\mathbf{9 5 \%}$ & $\mathbf{5 3 4}$ & $\mathbf{2 4 2}$ & $\mathbf{7 6}$ \\
\hline $\mathbf{9 0 \%}$ & 457 & $\mathbf{1 8 5}$ & $\mathbf{5 5}$ \\
\hline $\mathbf{8 5 \%}$ & $\mathbf{3 9 8}$ & $\mathbf{1 4 9}$ & $\mathbf{4 3}$ \\
\hline $\mathbf{8 0 \%}$ & $\mathbf{3 4 6}$ & $\mathbf{1 2 2}$ & $\mathbf{3 4}$ \\
\hline
\end{tabular}


Una vez obtenidos los tamaños de muestra necesarios, se solicitaron los marcos muestrales a la universidad, es decir, los listados de todos los estudiantes y docentes que pertenecen a la población objetivo, esto, para dar conciencia de que cualquier estudiante podía ser seleccionado y así cumplir con los requisitos de una muestra probabilista, la cual garantiza la fiabilidad de los resultados.

La selección de la muestra se realizó por medio de muestreo aleatorio simple sin remplazo, y a cada uno de los elementos pertenecientes a ella se les envió el instrumento mediante la plataforma Google Drive.

\section{Resultados y discusión}

A continuación se presentan los hallazgos obtenidos, de acuerdo con cada una de las preguntas en relación con la dimensión de capital social estructural obtenidos en el estudio relacionado en el contexto de la Universidad ECCI. Aquí se plantea un análisis comparativo de percepción entre estudiantes y docentes, lo que permitirá posteriormente plantear unas conclusiones sobre cada actor en relación con el otro.

Para este momento, se utilizó la Prueba Exacta de Fisher, valor $\mathrm{p}$ o valor de probabilidad. Por lo cual, los investigadores determinaron aceptar un margen de error en la hipótesis $<=$ 0.05. Dicho de otra manera, la hipótesis se validó con un $95 \%$ de confiabilidad.

Ahora bien, se procedió a aplicar la metodología del valor $\mathrm{p}$, con toda la rigurosidad que amerita una investigación científica. Por ende, se calculó la media, la varianza, la desviación estándar y la interpretación de la desviación estándar para hallar finalmente el valor $\mathrm{p}$. Lo cual permitió comprobar la veracidad de la hipótesis, en tanto el valor $p$ en los estudiantes fue de 0.04 y en los docentes de 0.05 Dicho de otra manera, se comprobó la exactitud de la hipótesis en un $95 \%$ y por consiguiente es una hipótesis válida.

Para una mayor claridad sobre los resultados, algunas de las preguntas que tienen relación directa se presentan de forma comparativa en un mismo gráfico, permitiendo así una mayor facilidad para la comprensión de los resultados.

El análisis se realizó en dos momentos; el primero, lo compone el análisis de correspondencias múltiples, para observar la existencia de asociación en cierta información que caracterice de manera correcta la población (se presentarán los mejores gráficos pues, en ocasiones, esta técnica no simplifica la situación, sino que por el contrario la dificulta un poco, perdiendo eficiencia); estos gráficos muestran información de manera muy resumida, y además es una gran herramienta para mirar asociación entre variables categóricas.

En segundo momento se presenta el análisis particular de cada pregunta por gráfico, comparando cada uno de los actores seleccionados para la investigación, con relación a la misma pregunta, lo que evidencia así los resultados particulares de cada ítem de la dimensión de capital social estructural.

Para el análisis de correspondencias múltiples, se tuvieron en cuenta las siguientes preguntas:

- ¿Su percepción sobre calidad en general en la Universidad, es más positiva hoy que hace un año? Percepción

- ¿En qué nivel de excelencia considera será valorado como profesional egresado de la Universidad en el contexto laboral? Valoración - ¿Qué nivel de pertenencia siente usted con respecto a la Universidad? Pertenencia.

El gráfico presenta una relación bastante clara, pues los estudiantes que tienen un sentido de pertenencia alto; también creen que la calidad ha mejorado en el último año y que su valoración como profesional egresado es alta. La misma situación está presente en el sentido de pertenencia medio, pues su percepción sobre a calidad también es media y cree que su valoración como profesional egresado de la institución también va a ser media. 
$\mathrm{Si}$ bien para respuestas de percepciones bajas de las tres preguntas se da la misma asociación, la verdad es que son muy aisladas, pues el estudiantado en general tiene una buena percepción sobre la institución, la cual podríamos calificar como media-alta.

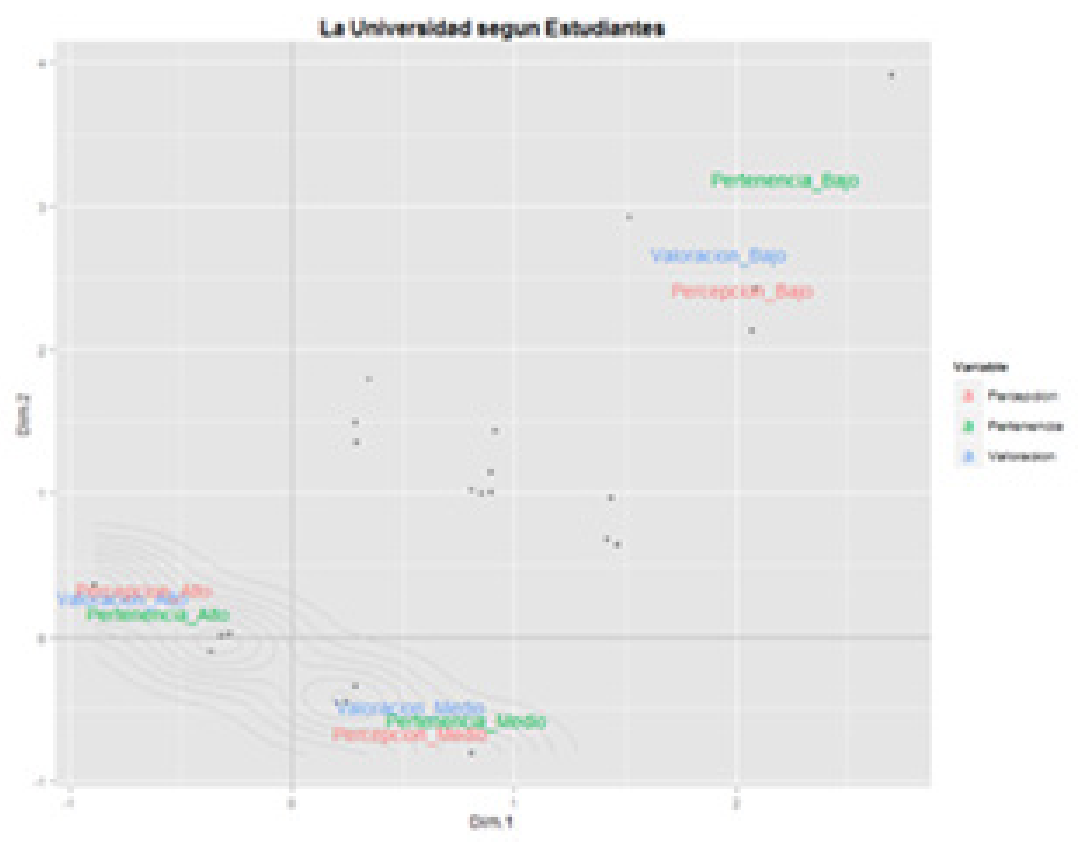

Es posible concluir entonces que la percepción de mejora que ha evidenciado la institución es mayor en los estudiantes que se encuentran más cercanos a la misma, es decir, el estudiante que está bien informado sobre la universidad, conoce mejor los puntos fuertes y por eso cree que la institución va por buen camino. Por tanto, la imagen de la universidad hacia los estudiantes, depende enteramente de cuanto exponga su imagen favorablemente hacia su estudiantado.

La situación docente es un poco diferente, si bien la relación entre las tres preguntas es la misma, no se da con la misma intensidad; hay mucha más dispersión al momento de asociar las tres preguntas. Se puede decir que la valoración como profesional egresado y la percepción de calidad, están muy asociadas, mientras que el sentido de pertenencia se hace un poco difícil de catalogar; por ejemplo, es más probable en un docente que su sentido de pertenencia con la institución sea alto pero la valoración como profesional docente y la percepción de la calidad sean medios, pues en los estudiantes, si se tiene sentido de pertenencia alto, es casi segura que la valoración como egresado y la percepción de calidad será alta. 


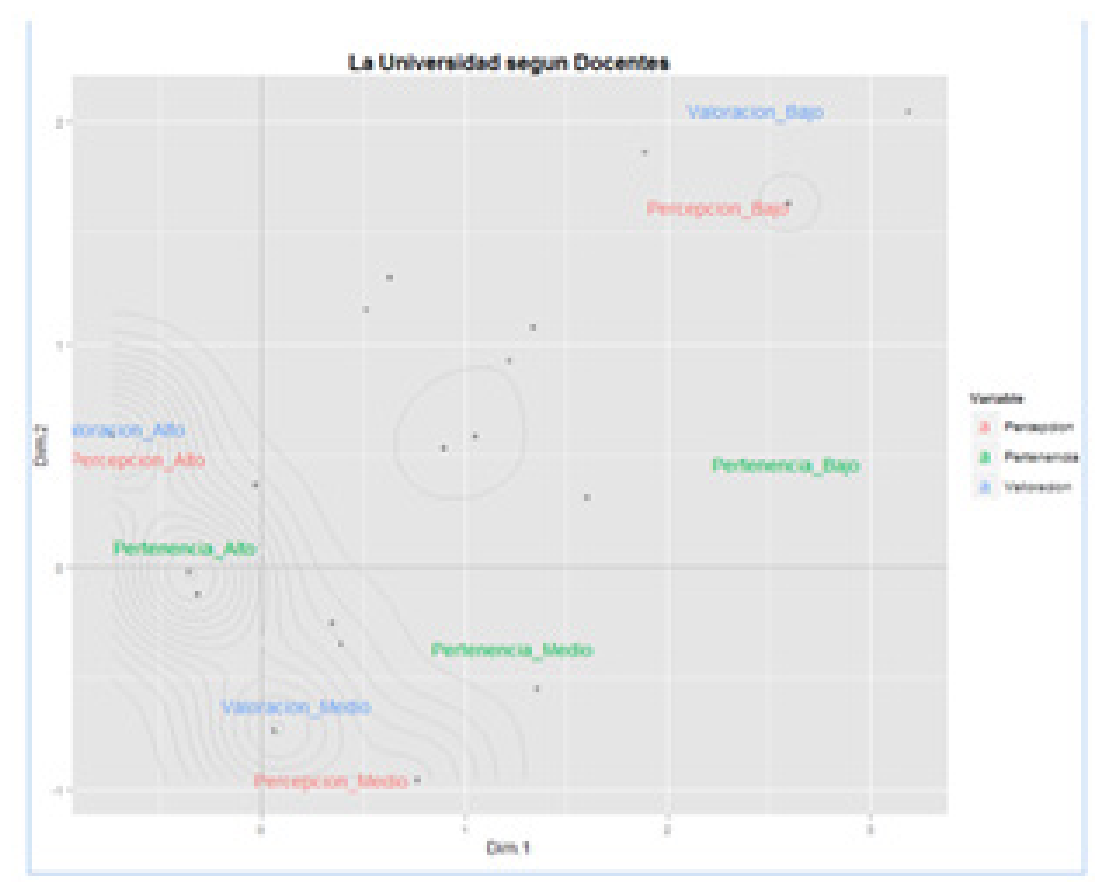

Todo esto puede ser por dos razones principales, la primera puede ser que el docente conoce mucho mejor la institución, por tener más contacto con aspectos administrativos y no tener una visión sesgada; o bien puede darse que sea un docente (que no tenga un horario definido) que no conozca de forma profunda la institución y sólo se limite a cumplir con su obligación contractual. En este caso, el nivel de información del encuestado es clave.

- ¿Su percepción sobre calidad en general en la Universidad, es más positiva hoy que hace un año? Percepción
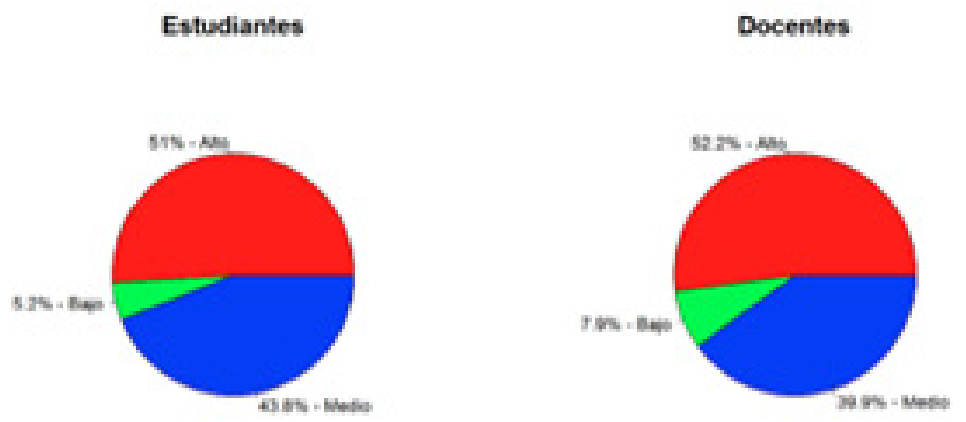
Se presenta una tendencia de comportamiento similar, entre la percepción de calidad alta que equivale a practicante la mitad de la muestra, tanto en estudiantes como docentes; sin embargo, los docentes son un poco más escépticos y marcan una tendencia más alta en el rango bajo. Esto significa que aunque la percepción de calidad supera la media es bastante desfavorable, pues el resultado evidencia a necesidad de fortalecer muchos procesos internos en la institución,

- ¿En qué nivel de excelencia considera será valorado como profesional egresado de la Universidad en el contexto laboral? Valoración

Estudiantes

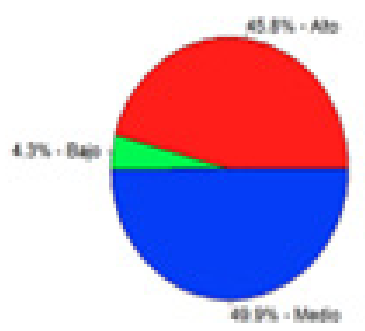

Los resultados de esta pregunta pueden presentar correspondencia en relación con los resultados del ítem anterior, ya que se evidencia una estrecha relación entre percepción de calidad y cómo se verán los egresados de la universidad en el contexto laboral externo; en este aspecto prima la percepción media, índice consecuente con los hallazgos obtenidos, resultados que pueden generar discusión con el planteamiento de Bourdieu, en relación con
Docentes

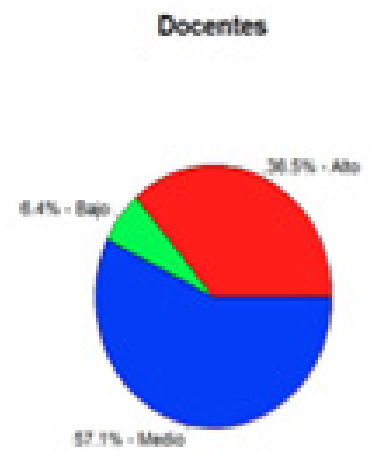

el capital social asumido como un constructo colectivo que se restringe a un contexto social y económico específico, en el cual los sujetos luchan por la obtención de un capital simbólico; en este aspecto, la comunidad académica de la Universidad ECCI reconoce la carencia de un stock en redes que le permita acceso a estos círculos donde puedan evidenciar sus capacidades y conocimientos.

Las siguientes preguntas se presentan comparativamente en el siguiente gráfico:

- ¿En su Universidad se evidencian oportunidades que permiten hacer parte de grupos que fomenten el desarrollo de proyectos a nivel extracurricular?

- ¿Conoce la normatividad que regula las relaciones internas de la Universidad?

- ¿Considera que la articulación con entes superiores de la Universidad es clara y fluye en el marco de las normatividad establecida? 

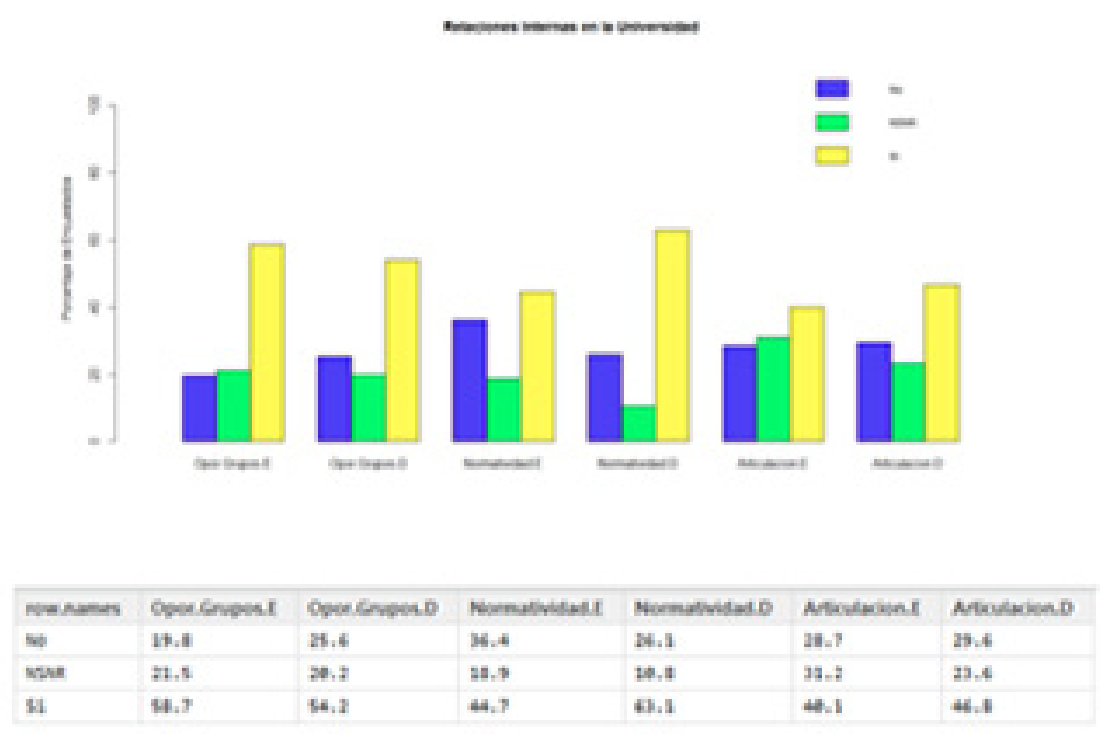

Frente al reconocimiento de oportunidades para consolidar redes y grupos asociativos se percibe una tendencia positiva, en promedio en la mitad de la población encuestada, con una leve diferencia entre estudiantes y docentes, donde los primeros con una ligera diferencia reconocen mayores oportunidades de pertenecer a grupos y desarrollar actividades para el logro conjunto de objetivos. En relación con los docentes, la menor percepción puede obedecer a que muchos profesores están vinculados por tiempo parcial a la institución y no conocen todas las opciones que existen de participar en redes.

En segundo lugar, el factor normativo, bastante relevante en la construcción de capital social resaltado por Bourdieu y teóricos como Coleman y Putnam, presenta un comportamiento algo atípico en este estudio, ya que a mayor pertenencia en redes por parte de los estudiantes, deberían evidenciar un mayor conocimiento de éstas; sin embargo, el estudio demuestra lo contrario, los docentes manifiestan mayor conocimiento de las normas internas de la institución; estos resultados entonces nos pueden llevar a identificar este factor como de alta incidencia en la construcción de capital social en la Universidad ECCI, ya que un grupo social que no reconozca sus obligaciones y derechos difícilmente puede llegar a establecer acuerdos que estén normalizados y regulados, y que moldeen su habitus como sujeto social.

Finalmente, con relación a las relaciones verticales en el contexto de la universidad, menos de la media en los dos actores estudiados reconocen que fluyen con claridad, factor que puede verse condicionado por falta de canales apropiados de comunicación o el contexto lleva a que la falta de conocimiento sobre la normatividad que restringe y posibilita dicha comunicación, la obstaculice. 
- ¿En qué grado identifica redes internas y externas en su Universidad que permita generar trabajo asociativo con otras universidades?

Estudiantes

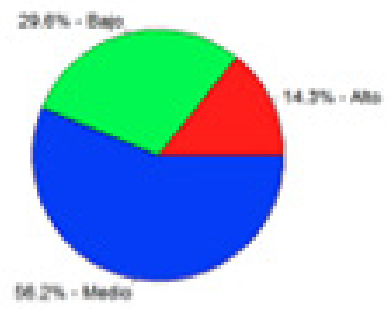

Docentes

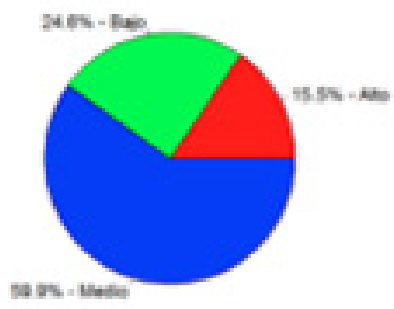

La comunidad académica señala para el desarrollo estratégico de funciones contundentemente, tanto estudiantes como como investigación y extensión, así como docentes que identifican redes externas a nivel el fortalecimiento de trabajo asociativo que medio es decir, pueden saber que existen pero fomente una mayor visibilidad institucional no conocen información sobre las mismas o no a nivel académico, afectando el acceso y pueden ser de su interés, frente a un porcentaje posicionamiento en diferentes estructuras bajo que sí las reconocen. Este factor se sociales. puede considerar como un elemento crítico

- ¿Se fomenta el liderazgo en los estudiantes que sobresalen en la Universidad a nivel académico e innovador?

Estudiantes

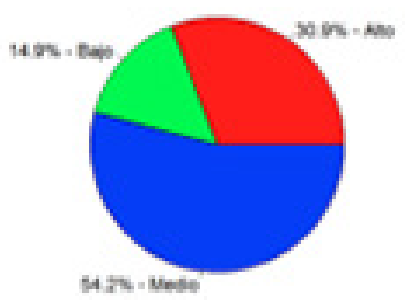

Docentes

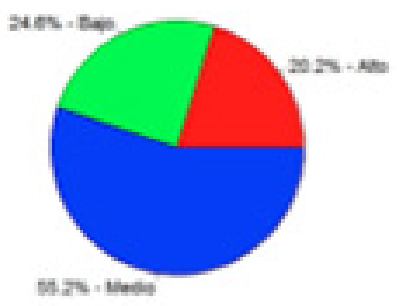


Un rango bajo-alto de estudiantes tienen tanto el nivel de pertenencia como el nivel la percepción de que en la universidad sí se de motivación individual del sujeto, pues a reconoce el liderazgo de los estudiantes, mayor reconocimiento mayor es la búsqueda comparado con un nivel mucho más bajo del capital simbólico en términos de Bourdieu de los docentes, cuya realidad condiciona en un círculo o estamento social definido.

- ¿Es invitado como estudiante/docente a participar en procesos de autoevaluación y mejoramiento a determinado nivel?

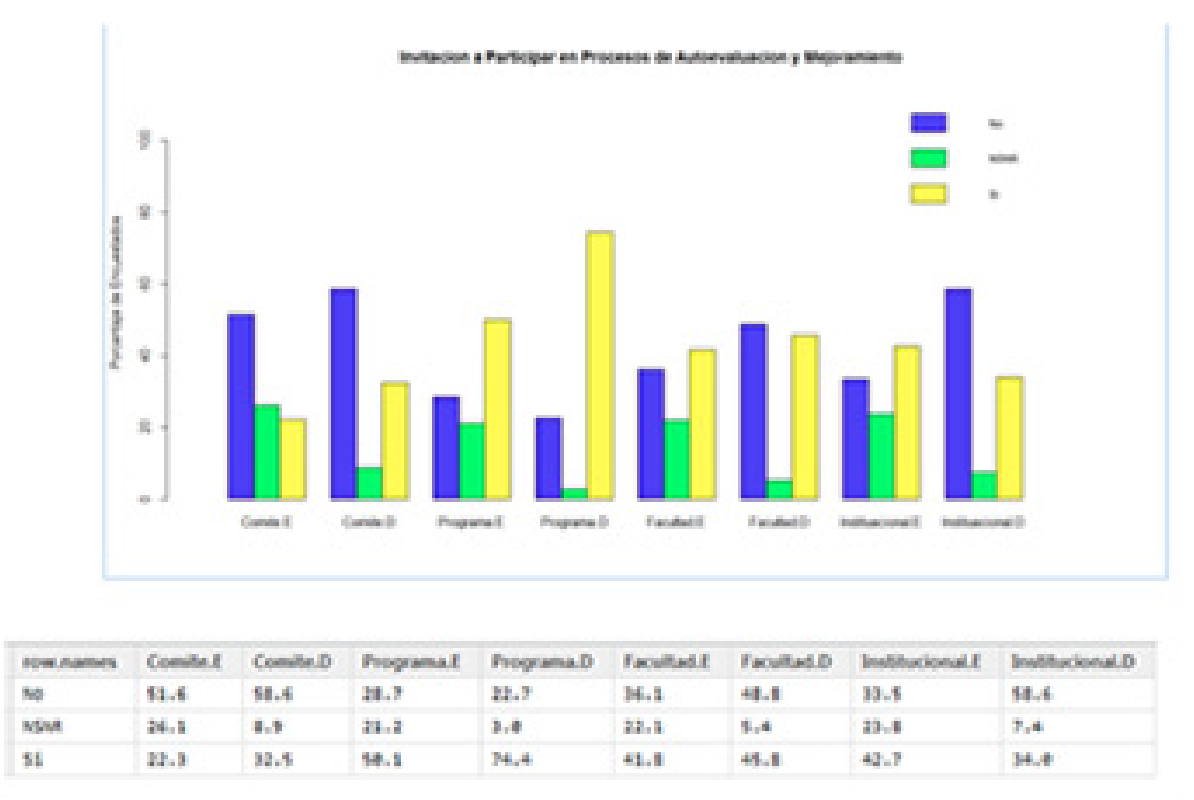

En este estudio se puede evidenciar que el mayor vínculo del docente con la Universidad es por medio de su programa académico, mientras que la media de los estudiantes demuestra que no son invitados a participar activamente en los diferentes cuerpos colegiados de la institución o no están interesados en participar, situación que con lleva a un menor grado de participación y, por ende, menor será el stock acumulado de capital social de la universidad. Pues como bien lo plantea Bourdieu, el capital social es un constructo colectivo normalizado.

Las siguientes preguntas se presentan comparativamente en el siguiente gráfico:

- ¿En qué nivel considera que la comunidad académica en general está dispuesta a hacer parte de redes asociativas?

- ¿En qué nivel considera que la Universidad está dispuesta a apoyar el desarrollo de redes asociativas? 


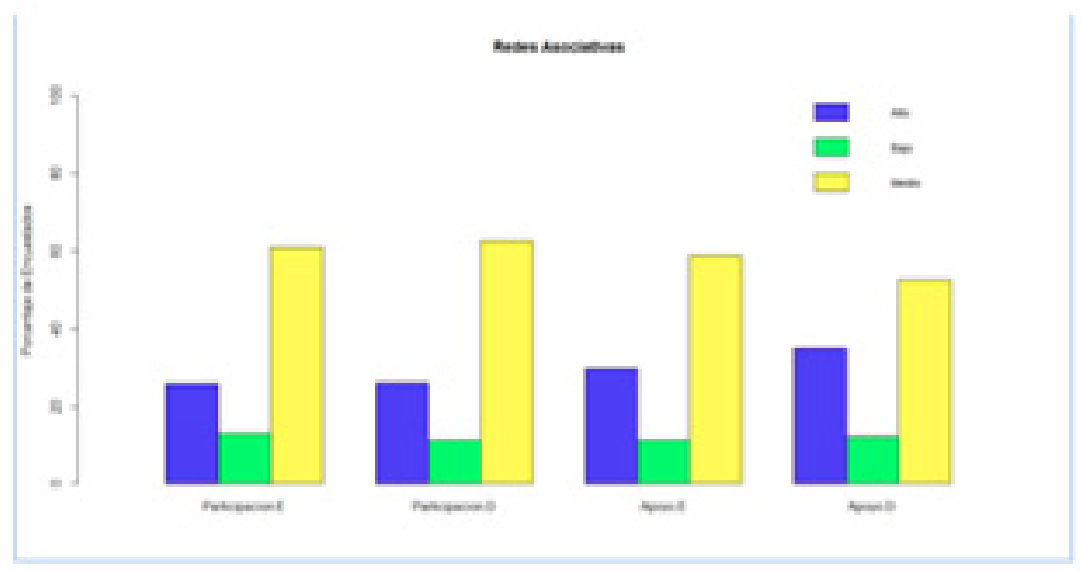

\begin{tabular}{|l|l|l|l|l|}
\hline row.names & Participacion.F & Participacion.D & Apoyo.E & Apoyo.D \\
\hline Alto & 25.8 & 26.1 & 29.8 & 35.6 \\
\hline Bajo & 13.2 & 11.3 & 11.5 & 12.3 \\
\hline Medio & 61.6 & 62.6 & 58.7 & 52.7 \\
\hline
\end{tabular}

Aquí se evidencia una baja intencionalidad relativa en general por participar en redes, cuya situación puede estar vinculada con la baja percepción que se tiene frente a la disposición de la universidad para apoyar la construcción en éstas. Otro factor que puede incidir es la falta de intencionalidad de reciprocidad y confianza de la comunidad académica frente a los resultados generados en conjunto con otros actores.

- ¿En qué nivel son efectivos los canales de comunicación en general en la Universidad?

Estudiantes

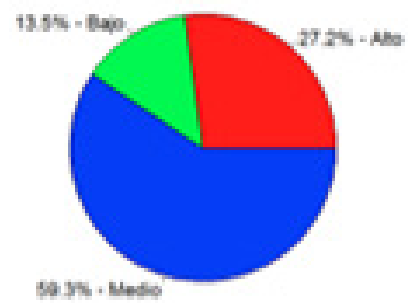

Docentes

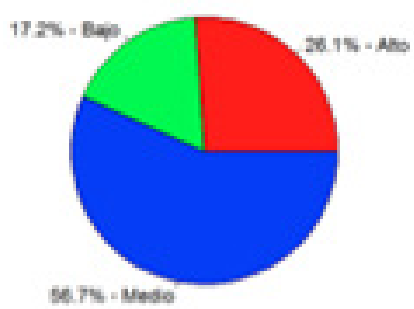


En esta instancia, prima una percepción media en los dos actores, de validar la efectividad de los canales de comunicación de la institución. En este aspecto, retomando las características del capital social estructural, es importante la comunicación efectiva para la construcción de confianza en la estructura social, lo que permite mayor sociabilidad $\mathrm{y}$, por ende, mejores resultados en los procesos generados desde la redes y la comunidad. En este sentido, la universidad requiere fortalecer sus estrategias en comunicación a nivel horizontal y vertical, asumiéndola como una mediación para el incremento de asociatividad y de capital social en la institución.

\section{Conclusiones}

Los resultados obtenidos luego de haber aplicado el valor $\mathrm{p}$ o valor de probabilidad a la encuesta, develan la veracidad de la hipótesis, en tanto el valor $\mathrm{p}$ se mantuvo en una escala de 0.04 a 0.05 ; estudiantes 0.049 y docentes 0.05 , respectivamente; en otras palabras la Educación Superior es el principal ente promotor de transformación en la sociedad, a partir del capital social, propósito central de este estudio.

Diferentes teóricos resaltan la importancia de las instituciones, la normatividad la educación, los valores y la cultura como agentes externos que afectan el habitus y el campo de los sujetos sociales que luchan por el capital simbólico, y se identifican a la vez como elementos que potencializan la generación de capital social con base en la asociatividad como trabajo colectivo y como fin, resaltando de esta forma la particularidad de la transdisciplinariedad del concepto.

La dimensión del capital social estructural se entonces relaciona con la interacción formal del sujeto con las organizaciones formales e informales en el contexto particular, restringido por las organizaciones del conjunto de estructuras estatales y no estatales a nivel local. Esta dimensión del capital social identifica variables que potencializan el desarrollo de redes, tales como formas, reglas y roles que permiten la consolidación de relaciones.

La concepción del concepto que plantea Pierre Bourdieu, evidencia cómo el capital social coexiste con el capital económico y el capital cultural, y se puede interpretar como fuente para que algunos individuos o clases sociales acumulen poder o lo transformen en diferentes tipos de capital. Esta capacidad de transformación genera efectos que no siempre benefician a toda una población sino a aquellos sujetos que están dentro del círculo de influencia del capital social; por ende, se puede afirmar que el capital social es excluyente, siendo ésta una de sus características más cuestionadas y calificada incluso como perversa.

Se evidencia asimismo que los niveles de percepción de capital social de la Universidad ECCI son bajos, pues dan cuenta de problemas estructurales internos entre los que se destacan, la falta de estrategias comunicativas a nivel de relaciones verticales principalmente, bajo nivel de reconocimiento del estamento normativo, factor que afecta las relaciones y posibilidades de asociatividad de la comunidad en redes endógenas, y una tendencia media a identificar fuentes de apoyo interno paras redes.

El estudio presenta una panorámica, la cual arroja tendencias de percepción no categóricas frente a la realidad, resultados que nos llevan a asumir como institución un complejo desafío relacionado con cómo se podría generar capital social en la Universidad ECCI, concepto comprendido como medio para fortalecer la comunidad sin afirmar tajantemente que el capital social sea la única alternativa, pero sí relevante frente al objetivo.

\section{Referencias}

Baranger, D. (2004). Epistemología y metodología de la obra de Pierre Bourdieu. Buenos Aires: Edición Prometeo.

Bourdieu, P. (1984). Espacio social y génesis 
de las clases . Buenos Aires: Nueva Visión. . (1988). La Distinción. Madrid: Taurus.

. (1997). Razones prácticas sobre la teoría de la acción. Barcelona: Editorial Anagrama.

. (2000). Poder, derecho y clases sociales. Bilbao: Desclée.

Champangne Patrick, Pinto Louis, Sapiro Gisel. (2007). Pierre Bourdieu Sociologo. Buenos Aires: Ediciones Nueva Visión.

Fernandez \& Ferrerias. (2009). La noción de campo en Kurt Lewin y Pierre Bourdieu. Un análisis comparativo. En: Revista española de investigaciones sociolólogicas No 127, 33-53.

Lenski, G. (1993). Poder y privilegio, teoria de la estratificación social . Barcelona: Paidos. Molina, X. (2008). La estructura y naturaleza del capital social en los organizaciones. Bilbao: Fundación BBVA.
Monroy, L. (2012). Análisis estadísticos de datos multivariados. Bogotá: Universidad Nacional de Colombia.

Richardson, J. (1986). Pierre Bourdieu "The social capital". En: Handbook of Theory and Research for the Sociology of Education, pp. 65-79.

Sapiro, G. (2007). "Pierre Bourdieu, el sociólogo". En: Pierre Bourdieu, el sociólogo (p. 19). Buenos Aires: Ediciones Nueva Visión.

Silvio, S. (2005). Fundamentos teóricos do capital social. Sao Paulo Chapecó: Editorial Argos.

Stavenhagen, R. (1991). Clases sociales y estratificación. México: Fondo de Cultura Económica.

Vázquez, F. (2002). La sociología como crítica de la razón. Madrid: Editorial Montesinos. 